\title{
Assessment-driven Learning through Serious Games: Guidance and Effective Outcomes
}

\author{
Othman Bakkali Yedri, Lotfi El Aachak, Mohamed Bouhorma \\ Computer Science, Systems and Telecommunication Laboratory (LIST), Faculty of Sciences and Technologies, \\ University Abdelmalek Essaadi Tangier, Morocco
}

\section{Article Info \\ Article history: \\ Received Sep 20, 2017 \\ Revised Jan 7, 2018 \\ Accepted Feb 2, 2018 \\ Keyword: \\ Assessment \\ E-assessment \\ E-learning \\ Game based learning \\ Learning outcomes \\ Serious games}

\begin{abstract}
Evaluation in serious games is an important aspect; it aims to evaluate the good transmission of pedagogical objectives, the performance of student in relation to these objectives defined in the pedagogical scenario, the content of the course and the predefined criteria. However, the effectiveness of learning is under-studied due to the complexity involved to gamify the assessment concept, particularly when it comes to intangible measures related to the progression of learning outcomes, which is among the most important aspects of evaluation in serious games. This paper reviews the literature regarding assessment due to their importance in the learning process with a detailed assessment plan applied on serious game. Then, it presents a framework used to facilitate the assessment design integrated in serious games. Finally, a significant example of how the proposed framework proved successful with corresponding results will conclude the paper.
\end{abstract}

Copyright (c) 2018 Institute of Advanced Engineering and Science. All rights reserved.

\section{Corresponding Author:}

Othman Bakkali Yedri,

Computer Science, Systems and Telecommunication Laboratory (LIST),

Faculty of Sciences and Technologies,

University Abdelmalek Essaadi Tangier, Morocco.

Email: othmanbakkali@gmail.com

\section{INTRODUCTION}

Over the last few years, serious games have been effective educational tools which attracted the attention of students, educators and professionals, used in many areas of knowledge including military defense, health care, manufacturing, education, economy, religion, business, emergency management, politics, city planning, scientific exploration, engineering, tourism and medicine [1]-[6], etc. For instance, this diversity it's not surprising because serious games can be designed to solve complex problems collaboratively [7], make business process more efficient, achieve predictive modeling and real time visualization [7], and more retention of knowledge compared to traditional methods. An example of educational decision is selection of courses in the higher education [8].

According to serious games definitions [9],[10],[11], we can resume the field in two term Fun and engagement. However, the serious side like learner assessment, recording user or learner data, enhancing results for professionals and so on presents a challenge for researchers according to their learning benefits and importance. For serious games prove their effectiveness; they must be able to transmit knowledge properly and allow measuring and analyzing the learning outcomes [12].

Traditionally talking, teachers or people in charge after every competence transfer they evaluate students learning achievement with exams. Besides, the field is used wisely in e-learning[13]-[16], and there are some generalized methods like assessment of student competence, assessment of student achievement. Assessment of learning and training requires a systematic approach to determine a person's achievements and areas of difficulty. Generally, assessment method is designed to evaluate many competencies but the final 
result is merely a grade without any description about student's achievement in every competency [17]. Standardized assessment methods often take less time and are easier to administer, and their results are readily interpretable [18]. However, this standardized assessment method are useless for non-computer or science person. Indeed, we provide to present an assessment framework which can be explicit and easy for professional and non-professional people.

In order to test the efficacy of this assessment plan, we will present our game entitled. Recruitment protocol that has as a purpose to train the player for a job interview. Obviously, the most important axis in the game is to judge the efficiency of learners and their abilities to adapt in the corresponding situation. A debriefing of the learner experience will be sent to the game tutor or an expert. The game is doted with some data mining algorithms. One of the challenges is to introduce an assessment plan in the game development process and face the instructors and experts how to evaluate the learning outcomes in order to identify students lacks for the given goal or field.

In this perspective of research we aim to present, first the literature review about assessment, eassessment their advantages, methods, tools and applications domains. Then, we discuss how to collect and to reuse data during the experience. In addition, we provide to present our framework by giving a significant example and we present the result of our proposal. Finally, we close by significant perspectives.

\section{LITERATURE REVIEW}

In our research of the assessment as scientific field, we find a number of definitions defining it, such as: 'An ongoing process aimed at understanding and improving student learning; It involves making our expectations explicit and public; setting appropriate criteria and high standards for learning quality; systematically gathering, analyzing, and interpreting evidence to determine how well performance matches those expectations and standards; and using the resulting information to document, explain, and improve performance' [15].

Also we find another definition that present the field 'as the systematic basis for making inferences about the learning and development of students; More specifically, assessment is the process of defining, selecting, designing, collecting, analyzing, interpreting, and using information to increase students' learning and development' [13]. According to these definitions, we can summarize assessment as a process aimed to understand, analyze, collect, interpret and use the information to improve learning and development of learners.

Generally speaking, assessment in an educational system comes in different aspects; Diagnostic assessment hopes to improve the learner's experiences. Is often used before teaching or at the beginning of the experience (Assess what the learner already know). Formative assessment Contributes to learning through providing feedback [19]. It occurs prior to and during the course and allows adjustments if necessary. It plays a regulatory role and learning support [20]. Summative assessment is like diagnostic assessment but it appears at theend of the learner experience. It's used to measures the learner's achievements at the end of the experiences. This measurement is often based on exams, papers projects and presentations, portfolios which help to submitting student progress and collecting information about the student. Integrative assessment or authentic assessment is a reflective and iterative participatory process that links knowledge (science) and action (policy) regarding complex global change issues [21]. Integrative tasks can be for formative or summative purposes.Especially for serious games, the formative assessment is the more useful and should be used in the game process to be part of the experience. Although several methods and techniques have been used to assess learning in serious games [22] and simulations in general, summative assessment is commonly accomplished with the use of pre- and post-testing, a common approach in educational research [23]. However, summative, formative, diagnostic and integrative assessment works together to improve learning. They should be the important components in the game design and at the game level.

In summary, assessment involves a rich infrastructure capabilities that enables us to evidence of student learning in a much deeper and often more authentic than what has been possible before with traditional methods. A number of software products are available for online educational testing and assessment [24]. However, the constructor can combine between a theory and technology to acquire desired result. In Table 1 we provide to present some of existing method and tools with corresponding description.

A literature review was performed to identify different approaches and theories in order to integrate assessment into serious games. In our research we find also a multitude of papers discussing the different types of assessment and papers discussing assessment frameworks for serious games. Some studies cover formative and summative assessment. For example authors in [28] uses serious game for formal assessment. However, the assessment model used in this study based on Gagne's nine events of instruction. Others , such as [29], are focused on learning skills and motivation which are assessed using a probabilistic approach performed by the user. Their approach is based on adaptability in order to help learners to increase their 
motivation skills. Indeed, providing an automated tool for monitoring and analyzing the actions performed by the learners, authors in [30] have as purpose to use petri nets extracted from the expert behavior in the game and compare the learner interactions to it using several diagnosis indicators.

Table 1. Assessment Methods and tools

\begin{tabular}{|c|c|}
\hline Type & Description \\
\hline $\begin{array}{l}\text { Assessment management } \\
\text { systems }\end{array}$ & Tools for instructors to administer and analyze tests \\
\hline $\begin{array}{c}\text { A Competence } \\
\text { Performance Analyzer }\end{array}$ & $\begin{array}{l}\text { Tool that keep trace of the learners' activity in the shape of events in } \\
\text { game and basing on these ones assesses the related performances respect } \\
\text { to a predefined set of competences[25]. }\end{array}$ \\
\hline $\begin{array}{l}\text { Tools for natural language } \\
\text { answer assessment }\end{array}$ & Tool that analyze and assess answers written in text area[14] \\
\hline Leap motion controller & A small device that can be connected to a computer using a USB[26]. \\
\hline Eye Tracking & $\begin{array}{c}\text { Eye Trackers measure the saccades (fast movements) and fixations } \\
\text { (dwell times) of human gaze[27] }\end{array}$ \\
\hline
\end{tabular}

Providing a list of theoretical questions, assessment matrix provides faculty members (especially ones with little assessment experience) with a structure for developing their assessment plan using a series of questions-as they answer the questions, they essentially articulate the plan [31]. Accompanying learners and their evaluation is a subject already treated in the literature[32], [33].The evaluation needs of serious games are particularly relevant to evaluation before apprenticeship, during apprenticeship (real-time help) and after apprenticeship (diagnosis of learned skills), possibly involving a debriefing between the learner and the monitor.

To summarize, all highlighted studies are generally focused on game play session, learner performance or achievement, summative and formative assessment and learning skills and motivation. Also, Assessment matrix is focused on a rhetorical side of assessment plan. However, it become necessary to combine several of these good techniques to encompass the strengths of all the assessment techniques reviewed. Especially for serious games assessment plan should combine between serious and fun in order to attempt the pedagogical objective, Assessment methods must be recognizable within the game design, as Michel and Chen [10] states "Serious games like every other tool of education must be able to show that the necessary learning has occurred.”.

\section{WHO'S IN CHARGE TO DESIGN AN ASSESSMENT MODEL FOR SERIOUS GAMES?}

Despite the abundance of literature on SGs, few papers provide a detailed description of the specific SG assessment design. Still, "many educational games do not properly translate knowledge, facts, and lessons into the language of games. This results in games that are often neither engaging nor educational" [34]. However, in this part of paper we provide to describe the roles of each author responsible of designing assessment in serious games. Persons who are in charge to design a serious game are: Professional expert, Pedagoge expert and Game designer expert.

\subsection{Professional expert:}

It is thus possible to reveal four criteria for measuring professional competence:

a. Learner level of expertise in a specific field,

b. A measure of his degree of knowledge in a given field,

c. The degree of operationally of the action he is carrying out,

d. The degree of variety of the reasoning he leads.

The Figure 1 describes the check list that should be done by the professional expert. As mentioned in the Figure 1, expert should:

a. Describe each situation and define their required competencies

b. Define the thematic with their level of competence

c. Classify action

d. Describe actions and different scenarios of each action 


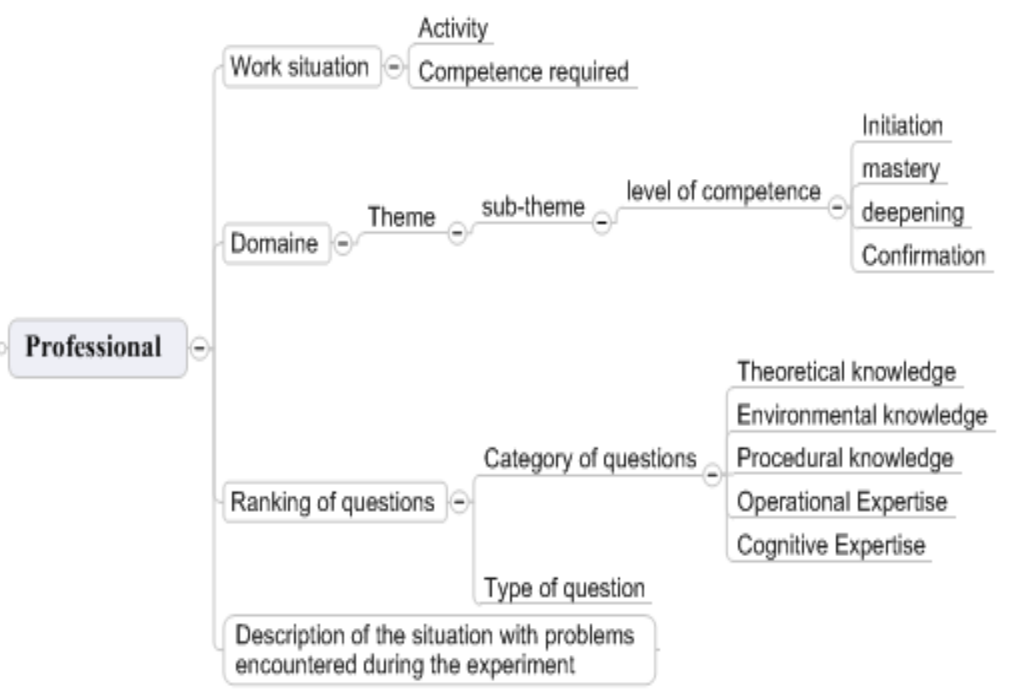

Figure 1. Professional expert

\subsection{Pedagogue Expert}

Is the person who's in charge to implement and to validate the learning transfer via the game experience. The Figure 2 describes the check list that should be done by the pedagogue expert. As mentioned in the Figure 2, pedagogue should:

Question: Action

Response: feedback (positive; negative)

As mentioned in the figure, pedagogue should:

a. Define the category of problem ( or question in quiz)

b. How to implement the problem in order to ensure better learning achievement

c. Define different errors types

d. When can be the guidance triggered. When each step can we implement this problem (pre in or post game)

e. Define educative message to show to the learner in order to complete his learning

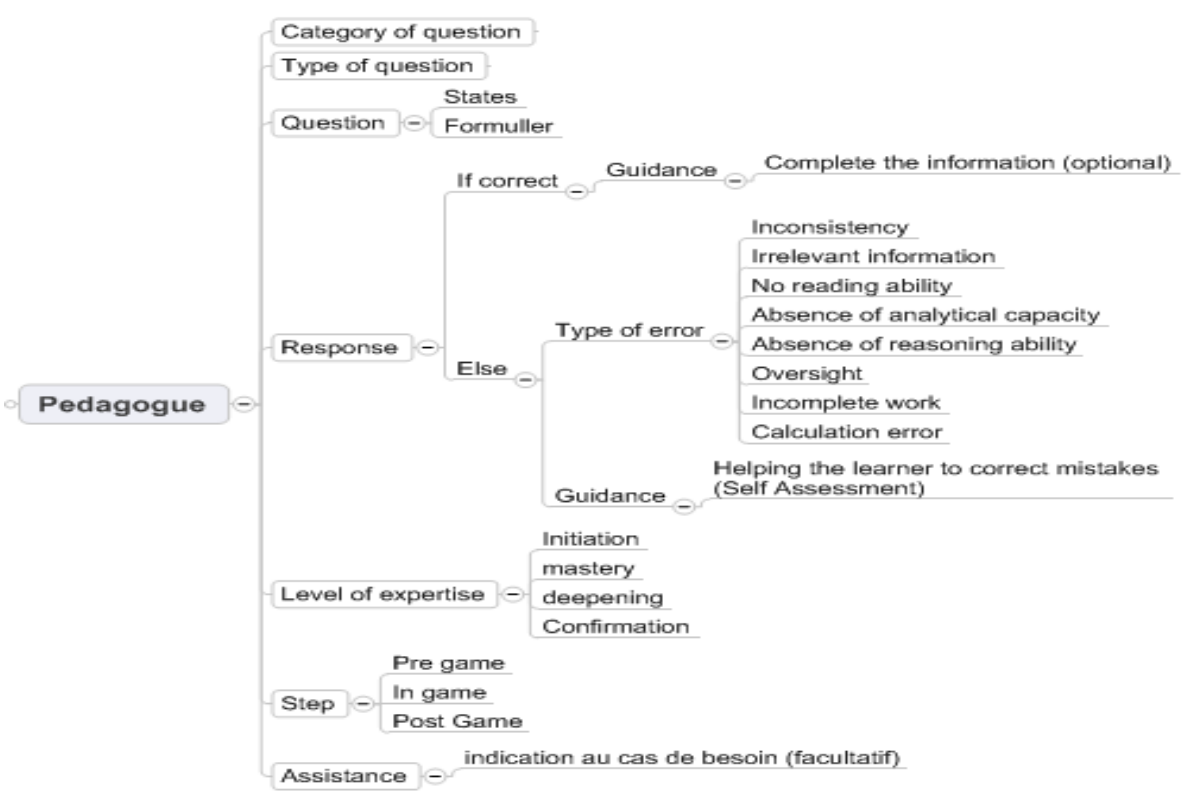

Figure 2. Pedagogue expert 


\subsection{Game Designer}

Game designer is the person who's in charge of gamifying instruction cited by both pedagogue and professional expert. He uses several tools and platforms for creating scenarios, object, sounds, and graphics and so on. Especially, for serious games game designer should have basics in different subject and pedagogy.

\section{ASSESSMENT PLAN DESIGN FOR SERIOUS GAMES}

In the light of our experience and according to the analysis made by our research team concerning the strong and weak point of each method, the method below, presents the proper integration of assessment plan in serious games design, supporting different methods and tools during the different steps of design and development process. The Table 2 sumarizes the main phases of development with their corresponding description.

Table 2. Development Phases

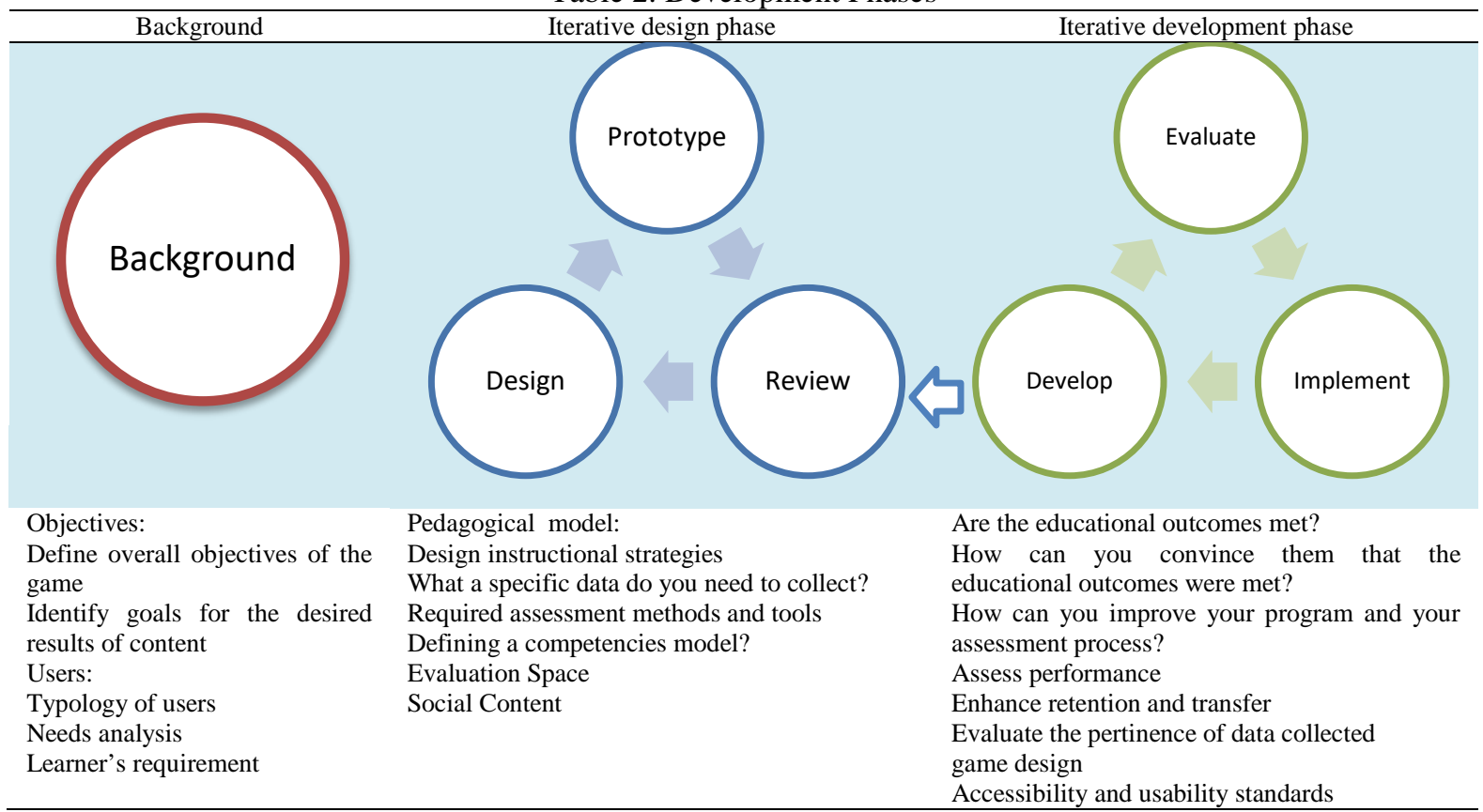

\subsection{Overall Objectives:}

Context layer: The expert or the game designer should situate the game in a specific context by defining domain, learning objectives, game intention, target audience and a brief description of summarizing the main objectives of the game as presented in Table 3.

Table 3. Overall Objectives

What are the overall objectives of the program?

Domain: Health, business, finance, military, management, recruitment, communication and marketing, business teaching, social security, Construction, Creative industries, life sciences, information and communications technology, Tourism, food and drink ...

Intention: Educative message broadcasting ,Informative message broadcasting , Marketing \& Communication message broadcasting

,Submit a subjective message ,Training ,Exchanging goods ,Tell a story ,Operating a license

Student requirement: what are the learner requirements?

Target Audience: Professionals, non-professionals, general population

Brief description: Text

\subsection{Learning Outcomes}

Learning layer: The expert or the game designer should have clear idea about learning outcomes. He or she must give a corresponding answer as presented below in Table 4 . 
Table 4. Educational Outcomes

What are the program's educational outcomes?

an ability to:

Engage in life-long learning;

Know the basics;

Able to memorize (factual learning) ;

sensor-motor skills;

Adapt and transfer knowledge in a new context, use information, methods, and theories in new situations;

Analysis of knowledge-based problem solving, prediction, projection conclusions, choice, argumentation.;

understanding the social environment and others;

Self-evaluation;

Others...

\subsection{Learner Portfolio:}

Students' experiences are collected throughout a program which is assessed by game engine using a predefined rules and a common scoring guide/stage. Portfolios may contain several informatione.g. "Research papers, reports, tests, exams, case studies, video, personal essays, journals, self-evaluations, exercises, etc". Portfolio is considered like a direct assessment method. Table 5 summarizes needed data to collect.

Table 5. Student Portfolio

student's portfolio

\begin{tabular}{l} 
student's portfolio \\
\hline Learner: Description \\
Type: Junior, Senior intermediate, expert \\
Tutor: Teacher, doctor, director... \\
Context: Open Space, Classroom, online... \\
Result: Score, time, number of correct answers, number of false answers, number of redundant faults \\
Note: Failed, medium, low, high, good, excellent \\
Decision: If we use serious games with e-learning platforms e.g.”moodle edx ...”, we can use students portfolio to complete data \\
information
\end{tabular}

\subsection{Assessment Methods:}

As presented by [9] learning objectives are brief statements that describe what the learner will be expected to learn by the end of the game. While educators or professionals use learning objectives in different ways to achieve a variety of instructional goals across multiple developmental stages, grade levels, age and so on. Each program will select the assessment methods as presented in Table 6 that will provide the most useful and relevant information. When selecting which assessment methods to use, consider the availability of resources, the usefulness of the results and the learning outcomes.

Table 6. Assessment Methods Needed to Collect Data

\begin{tabular}{|c|c|}
\hline \multicolumn{2}{|r|}{ What assessment methods will you use to collect data? } \\
\hline Method & Description \\
\hline Alumni Survey & $\begin{array}{l}\text { Can provide information about program satisfaction, preparation, employment status, skills for success. } \\
\text { At the end of the game } \\
\text { Type: Indirect }\end{array}$ \\
\hline Capstone Project & $\begin{array}{l}\text { Has as purpose to integrate knowledge, concepts, and skills acquiredby learner or the learner during the sequences } \\
\text { of the game. } \\
\text { In the game } \\
\text { Type: Direct }\end{array}$ \\
\hline Certification & $\begin{array}{l}\text { These standardized tests are developed by outside, professional organization to assess general knowledge in a } \\
\text { discipline } \\
\text { Type: Direct }\end{array}$ \\
\hline Employer Survey & $\begin{array}{l}\text { Information to collect can include on the job skills, field specific information, etc. } \\
\text { Type: Indirect }\end{array}$ \\
\hline Institutional Data & $\begin{array}{l}\text { Review both serious game and student data that is collected. } \\
\text { Type: Indirect }\end{array}$ \\
\hline Observations & Provide information on student behaviors and attitudes. \\
\hline Pre and post tests & $\begin{array}{l}\text { Typically an serious game is administered at the beginning and at the end of a course or program in order to } \\
\text { determine the progress of student learning } \\
\text { Type: Direct }\end{array}$ \\
\hline Game Review & $\begin{array}{l}\text { Reviewing a game involves determining if the objective is meeting the goals and outcomes that have been } \\
\text { established } \\
\text { Type: Indirect }\end{array}$ \\
\hline
\end{tabular}




\subsection{Data Collection}

Data collection is used to organize institutional processes, analyze student learning gaps and implement strategies, allocate resources, and continuously evaluates the efficacy of the learning objectives to support and improve student learning. It presents a quantitative data collection that helps to evaluate the learner experience. However, these data are collected from Patients; Students, learners, Teacher or the former; Evaluator agent; Guidance agent. Finally, the game designer should describe detailed needed data from each actor. All collected data depends to assessment method chosen by the game designer. Generally speaking they can be resumed into three layers: Pre-game, In-game, Post-Game. There are many reasons to collect data such as learners guidance during the game, test learner efficacy, measure the learner achievement; enhance the student level, improve the level of learner, recruitment; health analysis and so on. Table 7 presents the main specific data needed to collect.

Table 7. Specific Data to Collect

What specific data do we need to collect?

Name; Age ; Sex;Time;Score; Number of gestures; Number of right answers; Number of wrong answers. ; Check if the learner need
assistance or help;
If the game require reflection
Number of redundant fault
right action, too early, too late, suboptimal, erroneous and missing
Others...
These data depends on the nature of the game and it's can be defined by expert or game designer

The evaluation of the learners requires the collection of information from different sources according to the role incarnated by the learner in the scenario. The information required for evaluation thus varies in type and nature (depending on the role played by the actor), and must be acquired from sources of knowledge or data distributed over different software components.

Interactions can be evaluated by the time elapsed between the detection of the problem and its resolution as well as the impacted zone. Each data required for evaluation can use a different model of representation, as well as a calculation of evaluation specified to produce a learning score to validate or not the learning objective of a scenario. This knowledge can be produced individually or collectively (interactions between actors and / or simulated players).

\subsection{Data Evaluation:}

In this section, we present how can we interpret data with respect to the existing methods as presented in Table 8. Data analytics is a science consisting of examining raw data, in order to draw conclusions from this information. It is used to verify theories or to refute existing models. In this perspective of study, is focused on the learner achievement. Data analysis is then used to determine if the systems in place effectively operate efficiently and achieve the learning outcomes goals.

Table 8. Interpret and Evaluate Data

\begin{tabular}{|c|c|}
\hline \multicolumn{2}{|r|}{ How will you interpret and evaluate the data? } \\
\hline Method & Description \\
\hline Statistics & $\begin{array}{l}\text { Is one branch of mathematical science studying the collection, analysis, interpretation, presentation, and } \\
\text { organization of data[35] }\end{array}$ \\
\hline Business intelligence & $\begin{array}{l}\text { "intelligence" encompasses IT solutions providing decision support with professional, chain-end, reports and } \\
\text { monitoring dashboards activities [36] }\end{array}$ \\
\hline Web analytics & Meaning the study of the impact of a website on its users[37]. \\
\hline Operational research & $\begin{array}{l}\text { A mathematical discipline that contract with advanced analytical methods to help deciders to make the better } \\
\text { decision }\end{array}$ \\
\hline $\begin{array}{l}\text { Artificial intelligence } \\
\text { and data mining }\end{array}$ & $\begin{array}{l}\text { Data mining is a new concept in education domain. This concept can be helpful as a work of bridge between } \\
\text { this lacks of knowledge [38]. It's the process of analyzing data from different perspectives and summarizing } \\
\text { the results as valuable information. It has been defined as the nontrivial process of identifying valid, potentially } \\
\text { useful, novel and ultimately understandable patterns in data [39]. }\end{array}$ \\
\hline $\begin{array}{l}\text { Social network } \\
\text { analysis }\end{array}$ & $\begin{array}{l}\text { Meaning the study of the impact of social networks on its users. It means also the study of collection, analysis, } \\
\text { interpretation, presentation, and organization of user's data in social networks[40] }\end{array}$ \\
\hline $\begin{array}{l}\text { Information } \\
\text { visualization }\end{array}$ & The study of (interactive) visual representations of abstract data to reinforce human cognition. \\
\hline Learning analytics & $\begin{array}{l}\text { The application fields of the learning analytics concern modeling of user knowledge, user behavior, and user } \\
\text { experience, user profiling; modeling of key concepts in a domain and modeling a domain's knowledge } \\
\text { components, and trend analysis }\end{array}$ \\
\hline
\end{tabular}




\subsection{Debriefing and Feedback}

After each programme, tutor or former should convince parent of learners that the learning objectives are achieved as presented in Table 9. Outcomes define what you want a serious game to accomplish. Generally speaking, learning outcomes statements may be considered to be exit behaviors. Outcomes achievement can be presented in post-game for student and tutor like a quiz to have more information and to help the game designer to enhance our game engine. Information about student learning outcomes assessment results will be available at the appropriate levels of granularity for use by programs, in analysis and evaluation, and decision-making.

Table 9. Learning Achievement

How can you convince them the objectives were met?

Goals :Attend the aims of a game

Outcomes:the changes in the lives of individuals, families, organizations, or the community as a result of the game. Evaluate intrinsic motivation

Indicators:the specific, measurable information collected to track whether an outcome has actually occurred

\section{REVERSE ENGINEERING}

When applying assessment plan in design process and if the designer respect the instruction as presented above the result will be as present in the Figure 3. Evaluation type in serious games: Real time evaluation: This evaluation relates to a diagnosis evolving throughout the game (game scenario). Real-time evaluation refers to the evaluation of an action or mission (Behavior space). To evaluate an action, the calculation of a score is based on the corresponding indicators.

Final individual evaluation: Individual evaluation is a summative evaluation that evaluates and certifies the learner's learning in the game scenario. This evaluation compares the state of the learner's model (learner actions As well as his/her knowledge) with the model of the domain and establishes a diagnosis on the skills acquired and the skills that remain to be learned.

Collective evaluation: Using the Social Space, an interaction graph is constructed, representing the different communications and interactions between the different actors (learners as well as simulated actors). With this graph, we know who contacted who, when, and for how long, as well as information exchanged. Combining the information from the different spaces, we have the possibility to deduce a causal link between the missions of the various actors (the actor failed in his mission because the actor B did not send the right information) and thus obtain a complete and precise evaluation of all the actors.

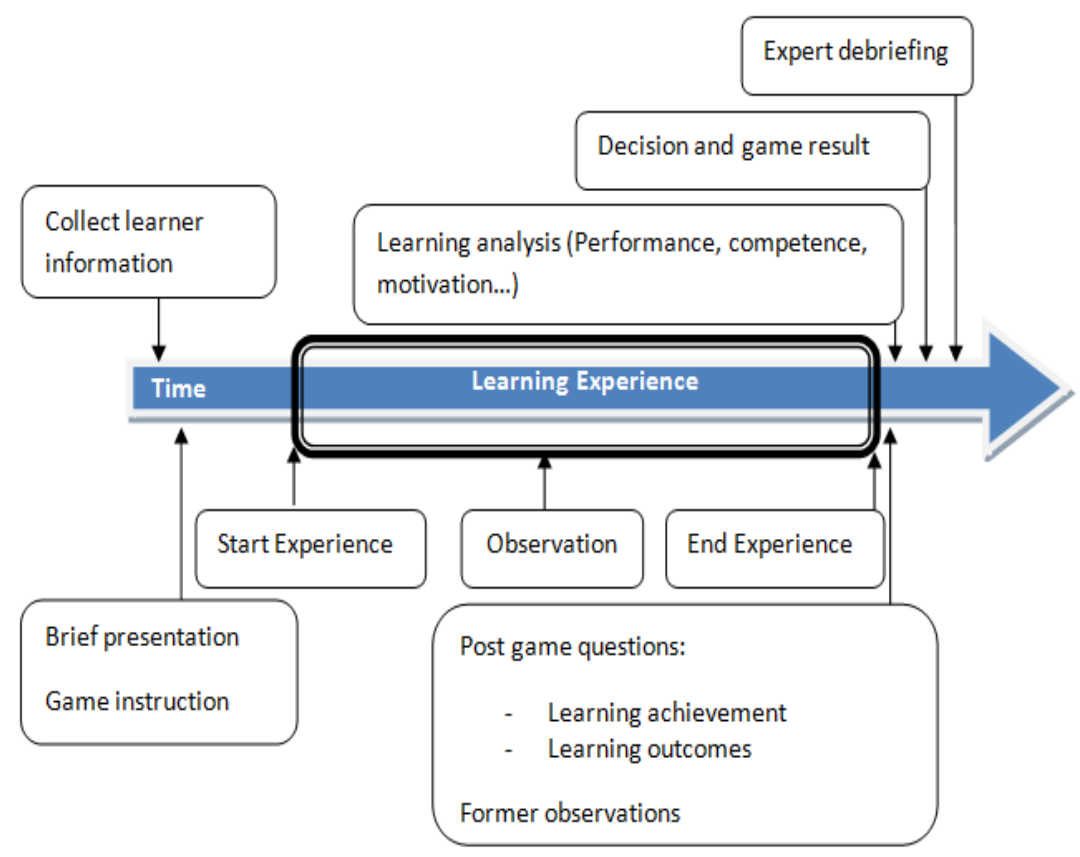

Figure 3. Learning timeline 
Global evaluation will be done at the end of the game play session. It will contain both individual and collective evaluation. Certification of a learner's skill or knowledge can be achieved through negotiation as presented in [41]. For Massive multi online games creators or game designers should take in consideration collaboration, culture and communication. At the end of each experience test pedagogue and professional expert respectively must evaluate output of game and student learning achievement. This operation is important for both to enhance assessment plan and to ensure homogeneity of collected data. However, this method is evaluated by implementing some games in our laboratory and by applying this method on some existing games as presented in the next part.

\section{PROOF OF CONCEPT}

\subsection{Existing Serious Games}

Mecagenius[5] is an example of serious game that uses in process assessment based on the monitoring of states. The purpose of the game is to train engineer in mechanics, providing them with a virtual laboratory and various tools. The learner has to give a diagnosis and a prescription to each case of study. He has the possibility of running different types of tests. The system also collects secondary information for more formative assessment.

Simventure[42]: is an example of business game. It allows the player's to manage a company, dealing with four major types of issues: production, organization, sales and market, and finance. It proposes also some additional activities, such as debriefing, answering questions, writing essays, and forecasting events and outcomes and business planning.

PIXELearning's Enterprise Game[43] is a similar business game. Learner can define a product meeting the market demand in terms of quality and price is the most important factor to make the business viable. Defining their proper marketing strategy is a key as well. It's allows to learner new aspect such as competition and collaboration.

\subsection{Result and Discussion}

The purpose of our game Recruitment protocol game as presented below in Figure 4 is to prepare the player as good as possible for his job interview or job promotion, providing him with an interview and various real situations. The learner should manage to face each situation by selecting right answers in the right moment. The player must choose among several answers the one that seems to him the most adapted, thus becoming aware of the rules of politeness, adaptability, motivation and so on, in order to be implemented during a job interview. The system also collects secondary information for more formative assessment.

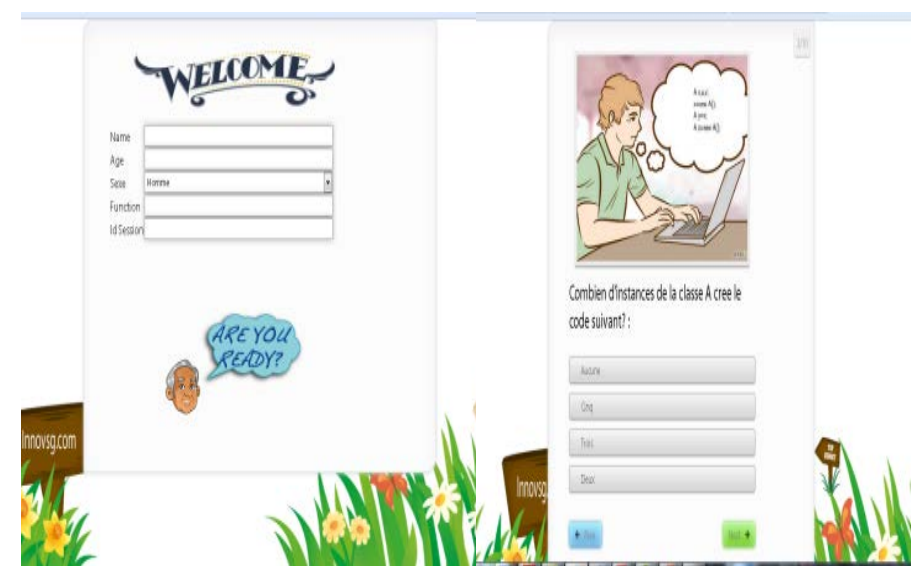

Figure 4. Recruitment protocol

The choice of recruitment is not arbitrary, because it combines theory and experience. Also, in the recruitment process we have all types of assessment, pre, in, post and global evaluation. The choice of a good element is not an easy job and it's required and number of techniques and rules that we would respect to achieve the desired results. The designing team (pedagogue, expert and designer) must define different scenarios, actions, rules, data to collect and so on. When designing and developing the game we were a team 
composed by a game designer, recruit expert and pedagogue. As mention above in our proposed assessment plan, actors defined the game objectives. However, the game subscribes in the recruitment and business domain. It has as intent Educative message broadcasting, Informative message broadcasting and training and it has professionals as a target.

The evaluation of the learners in recruitment protocol requires the collection of information from different sources according to the role incarnated by the learner in the scenario. However, the expert and pedagogue have classified question into four groups, in each group of question there are several question with their corresponding result, choices, guidance, difficulty and so on. In the Table 10 we present a part of proposed question.

Table 10. Quiz

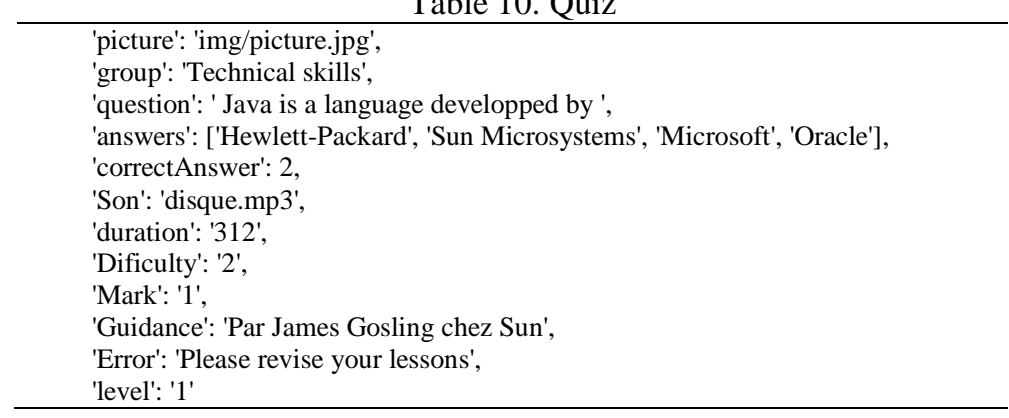

The main objective of the game is to train new bachelor for a job interview and to help them to develop their skills required for specific jobs. The game is for computer and science student. In Table 11 we present student's portfolio required in recruitment protocole. In order to achieve optimal result, we provide to collect a number of data as presented in the table below in Table 12. Collected Data

Table 11. Student's Portfolio: case of Recruitment Protocole

\begin{tabular}{l}
\hline Learner: is a student or employee \\
Type: Junior, Senior intermediate, expert \\
Tutor: Teacher, project manager \\
Context: online \\
Result: Score, time, number of correct answers, number of false answers, number of redundant faults, result by skills \\
Note: Failed, medium, low, high, good, excellent \\
Decision: pass to the next level, repeat the stage
\end{tabular}

Table 12. Collected Data

What specific data do we need to collect?

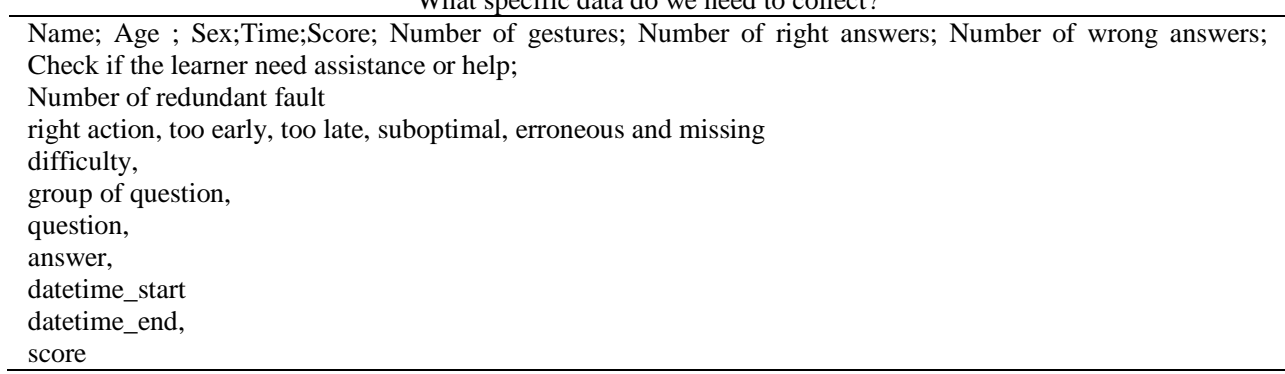

The pedagogue expert requires using the evaluation method capstone project considering the large number of advantages that it brings and it is perfectly appropriate with the nature of our project. This method has as purpose to integrate knowledge, concepts, and skills acquired by learner during the sequences of the game. Statistics and learning analytics are applied to measure, collect, analysing and reporting of data about learners and their contexts, for purposes of understanding and optimizing learning and the environments in which it occurs.

To facilate the monitoring to the player status, there is a controle panel that allows the activation and/or monitoring of the following actions: enable/disable the sound, to show a number of errors, to controle 
a number of questions in each level, to contrôle a time spent in each action, display learning debriefing at the end of the experience. After the players answer it, the game provides a feedback starting whether they missed the issue and if they spent more or less than the estimated time also if they miss a stage or some question an alert will be triggered. A player can demand assistance as soon as possible. The game was evaluated for a computer science class composed of 34 students who, after playing the game they have a detailed result about their experience and a debriefing about their lacks and how to develop it. Also the tutor, have a dashboard from them we extract the chart in the Figure 5. Especially, corporate sectors need some specific skills to enhance in the game or learning experience:

a. Technical Skills: Operational the necessary skills to produce the product or service Supplies/Raw Materials to obtain optimal result.

b. Managerial Skills: Management planning, organizing, supervising, directing, networking Marketing/Sales identifying customers, distribution channels, supply chain Financial managing financial resources, accounting, budgeting Legal organization form, risk management, privacy and security Administrative people relations, advisory board relations Higher-order thinking skills

c. Entrepreneurial Skills: Business Concept business plan, presentation skills Environmental Scanning recognize market gap, identify user needs, exploit market opportunity Advisory Board and Networking balance independence with seeking assistance

d. Personal Maturity Skills: Self-Awareness ability to reflect and be introspective Accountability ability to take responsibility for resolving a problem Emotional Coping emotional ability to cope with a problem Creativity ability to produce a creative solution to a problem Risk taking, tolerance for frustration. After putting the game into experience we obtained this global result.

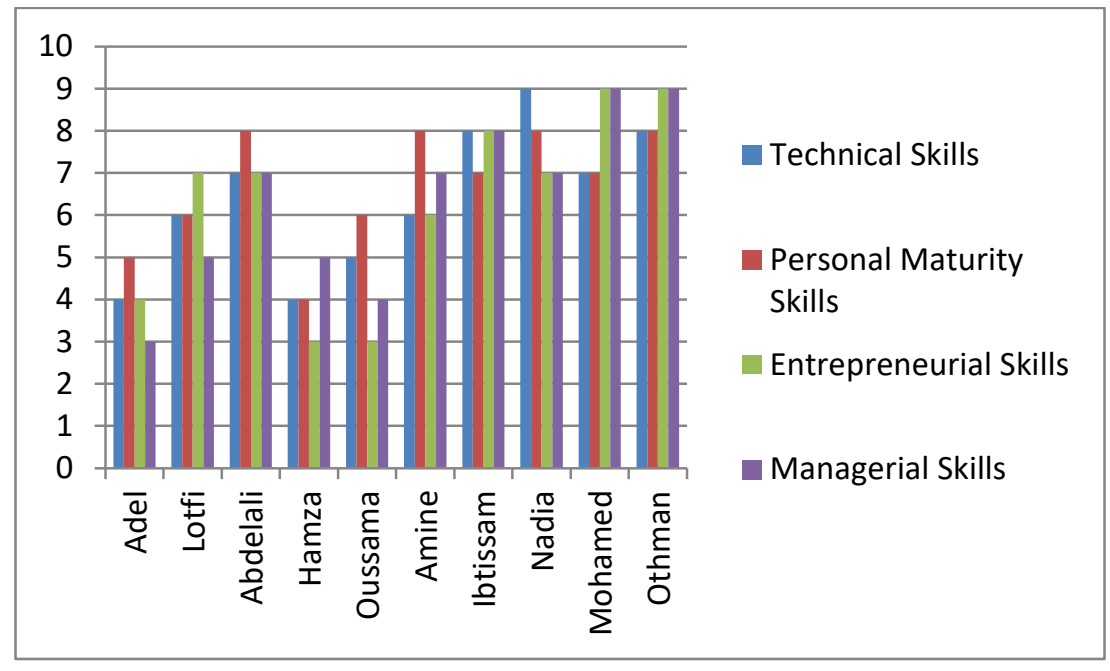

Figure 5. Learning experience result

The questions are grouped into four skills group technical skills, personal maturity skills, entrepreneurial and managerial skills. The chart presents the result of player's experience. According to obtained result, the former or the company has a visibility to their employee's competencies. Also, the learner, trainee or employee has a clear idea about their lack and skills to develop to have better career evolution. The results as presented in the Figure 5 shows that the majority of players have a good level in technical skills and personal maturity skills but they still have to improve others skills.

At the moment, this assessment strategy proved successful. We hope to test it in a large wide to validate it and to ensure better integration of assessment in the future for both the professional and nonprofessional. The main objective of an evaluation plan is to evaluate, in general terms, the viability of all pedagogical aspects of a serious game, including the description and analysis of development prospects. Regardless of the size of the project, the evaluation plan is an essential step for anyone seriously wanting to create their own serious game. As this step is too often overlooked, our research team has decided to simplify things by proposing a simple and effective structure to build an evaluation plan tailored to the needs of the designer. 
According to this study we can define easily needed steps and parameters to integrate assessment in the game design process, in order to create a beneficial strategy of assessment that will help the learners to improve their level and accomplish their learning achievement, in addition it will make the serious game one of the great tools for learning assessment.

This project is a special device to accompany the creators of the game, from the stage of preparation of the story and preparation of the project until the end of preparation of an evaluation plan. This project was designed to help decision-makers or educators ensure the sustainability of their serious games. Highlight of this approach are:

a. The simplicity of expressing needs.

b. The framework can be exploitable by both for professional and non-professional people

c. The possibility of integrating the elements of quantitative and qualitative evaluation

d. Gives a clear and detailed idea of the project

In this step of our project we provide to collect the necessary information about the evaluation plan. As a first step, we have integrated the quantitative evaluation considering the different advantages that they present. In future work, we will integrate assessment intangible measures as the motivation, behavioral changes, and learner performance.

\section{CONCLUSION AND FUTURE WORKS}

The assessment in serious games is one of the important mechanisms that push learners to achieve their learning objectives; therefore, it makes the serious games one of the effective learning tools. During this paper, several assessments techniques and methods applied on serious games have been discussed. Among the techniques used by our research team there are the matrix of assessment and data collection, they have been applied on some kinds in the game developed by our team of research recruiting protocol, according to this experience a new scheme for a proper integration of assessment in serious games, supporting different methods and tools in different steps of development process has been developed. The proposed schema will be improved thereafter, in order, to enhance the platform that will help the game designer to establish some assessment strategies in a very simple way, respecting the acquired and the learning outcomes envisaged by the experts.

\section{REFERENCES}

[1] V. Wattanasoontorn, I. Boada, R. García, and M. Sbert, “Serious games for health,” Entertain. Comput., vol. 4, no. 4, pp. 231-247, Dec. 2013.

[2] B. M. C. B. G. 09/01/09 20:07, ““America's Army' : le jeu comme levier de recrutement.”[Online]. Available: http://www.journaldunet.com/ebusiness/crm-marketing/selection/dix-serious-games-marketing-originaux/america-sarmy-le-jeu-comme-levier-de-recrutement.shtml. [Accessed: 08-Oct-2015].

[3] N. Baldissin, S. Bettiol, S. Magrin, and F. Nonino, Business game-based learning in management education. Lulu.com, 2013.

[4] K. Huotari and J. Hamari, “Gamification” from the perspective of service marketing," in Proc. CHI 2011 Workshop Gamification, 2011.

[5] “Un serious game en génie mécanique pour découvrir, apprendre, fabriquer | mecagenius.univ-jfc.fr.” [Online]. Available: http://mecagenius.univ-jfc.fr/. [Accessed: 22-Feb-2016].

[6] E. Pittet and M. Rossi, "La gamification et les RH," 2016.

[7] "IBM Serious Games Solutions - Smarter Serious Gaming - United States." [Online]. Available: https://www.ibm.com/services/us/gbs/gaming/. [Accessed: 15-Aug-2017].

[8] M. Sudarma, A. A. K. Oka, and I. Cahya, "Decision Support System for the Selection of Courses in the Higher Education using the Method of Elimination Et Choix Tranduit La Realite,” Int. J. Electr. Comput. Eng., vol. 5, no. 1, p. 129, 2015.

[9] M. Zyda, "From visual simulation to virtual reality to games,” Computer, vol. 38, no. 9, pp. 25-32, 2005.

[10] D. R. Michael and S. Chen, Serious Games: Games that Educate, Train and Inform. Thomson Course Technology, 2006.

[11] C. C. Abt, Serious games. Lanham, MD: University Press of America, 1987.

[12] L. Elaachak, A. Belahbibe, and M. Bouhorma, "Towards a System of Guidance, Assistance and Learning Analytics Based on Multi Agent System Applied on Serious Games,” Int. J. Electr. Comput. Eng. IJECE, vol. 5, no. 2, pp. 344-354, 2015.

[13] M. J. Bresciani, C. L. Zelna, and J. A. Anderson, “Assessing student learning and development,” Handb. Pract. U. S. NASPA, 2004.

[14] L. CUTRONE, “ASSESSING STUDENTS’ANSWERS TO OPEN QUESTIONS,” ATHABASCA UNIVERSITY, 2010.

[15] K. Swan, J. Shen, and S. R. Hiltz, “Assessment and collaboration in online learning,” J. Asynchronous Learn. Netw., vol. 10, no. 1, pp. 45-62, 2006. 
[16] C. A. Palomba and T. W. Banta, Assessment essentials: planning, implementing, and improving assessment in higher education, 1st ed. San Francisco: Jossey-Bass Publishers, 1999.

[17] S. Andayani, S. Hartati, R. Wardoyo, and D. Mardapi, "Decision-Making Model for Student Assessment by Unifying Numerical and Linguistic Data,” Int. J. Electr. Comput. Eng. IJECE, vol. 7, no. 1, p. 363, Feb. 2017.

[18] M. M. Clarke, G. F. Madaus, C. L. Horn, and M. A. Ramos, "Retrospective on educational testing and assessment in the 20th century," J. Curric. Stud., vol. 32, no. 2, pp. 159-181, Mar. 2000.

[19] D. Robinson, "University of [Online]. Available: https://www.exeter.ac.uk/staff/development/academic/assessmentandfeedback/principlesofassessment/typesofassess ment-definitions/. [Accessed: 27-Mar-2016].

[20] C. Boston, “The Concept of Formative Assessment. ERIC Digest.,” 2002.

[21] R. E. Munn, M. C. MacCracken, and J. S. Perry, The Earth system: physical and chemical dimensions of global environmental change. Chichester: Wiley, 2002.

[22] C. S. Loh, A. Anantachai, J. Byun, and J. Lenox, “Assessing what players learned in serious games: in situ data collection, information trails, and quantitative analysis," in 10th International Conference on Computer Games: AI, Animation, Mobile, Educational \& Serious Games (CGAMES 2007), 2007, pp. 25-28.

[23] K. Becker and J. R. Parker, The Guide to Computer Simulations and Games. John Wiley \& Sons, 2011.

[24] “ONLINE TESTING | assessmentfocus.com.” [Online]. Available: http://www.assessmentfocus.com/onlinetesting.php. [Accessed: 28-Feb-2017].

[25] E. Parodi and M. Vannucci, “A Competence Performance Analyser Tool for Assessing Players' Activity in Serious Games,” Pedagog.-Driven Serious Games PDSG 2012, 2012.

[26] “Leap Motion,” Leap Motion. [Online]. Available: http://leapmotion.com/. [Accessed: 28-Mar-2017].

[27] L. E. Nacke, A. Drachen, and S. Göbel, "Methods for evaluating gameplay experience in a serious gaming context," Int. J. Comput. Sci. Sport, vol. 9, no. 2, pp. 1-12, 2010.

[28] B. Meyer, ECGBL2009- 4th European Conference on Games-Based Learning: ECGBL 2009. Academic Conferences Limited, 2010.

[29] M. D. Kickmeier-Rust, E. Mattheiss, C. Steiner, and D. Albert, "A Psycho-Pedagogical Framework for MultiAdaptive Educational Games:,” Int. J. Game-Based Learn., vol. 1, no. 1, pp. 45-58, 2011.

[30] P. Thomas, J.-M. Labat, M. Muratet, and A. Yessad, "How to Evaluate Competencies in Game-Based Learning Systems Automatically?,” in Intelligent Tutoring Systems, vol. 7315, S. A. Cerri, W. J. Clancey, G. Papadourakis, and K. Panourgia, Eds. Berlin, Heidelberg: Springer Berlin Heidelberg, 2012, pp. 168-173.

[31] B. M. Olds, “Effective Strategies to Assess the Impact of e-learning,” 2002.

[32] D. Lourdeaux, "Réalité Virtuelle et Formation : Conception d'Environnements Virtuels Pédagogiques," PhD Thesis, 2001.

[33] W. L. Johnson, J. Rickel, R. Stiles, and A. Munro, "Integrating Pedagogical Agents into Virtual Environments," Presence Teleoperators Virtual Environ., vol. 7, no. 6, pp. 523-546, Dec. 1998.

[34] F. Bellotti, R. Berta, and A. De Gloria, "Designing Effective Serious Games: Opportunities and Challenges for Research,” Int. J. Emerg. Technol. Learn. IJET, vol. 5, no. SI3, Nov. 2010.

[35] Y. Dodge, Ed., The Oxford dictionary of statistical terms, First published in paperback 2006. Oxford: Oxford Univ. Press, 2006.

[36] king-theme.com, “\#BusinessIntelligence: for a better Control of Data.” .

[37] "What is Web analytics? Webopedia Definition." [Online]. Available: http://www.webopedia.com/TERM/W/Web_analytics.html. [Accessed: 28-Mar-2017].

[38] S. K. Verma and R. S. Thakur, "Fuzzy Association Rule Mining based Model to Predict Students’ Performance," Int. J. Electr. Comput. Eng. IJECE, vol. 7, no. 4, p. 2223, Aug. 2017.

[39] H. Dai, R. Srikant, and C. Zhang, Advances in Knowledge Discovery and Data Mining: 8th Pacific-Asia Conference, PAKDD 2004, Sydney, Australia, May 26-28, 2004, Proceedings, vol. 3056. Springer, 2004.

[40] E. Otte and R. Rousseau, "Social network analysis: a powerful strategy, also for the information sciences," J. Inf. Sci., vol. 28, no. 6, pp. 441-453, Dec. 2002.

[41] A. Chadli, E. Tranvouez, and F. Bendella, "Cooperative skills evaluation and participative multiagent simulation for rodent control training,” 2010, pp. 41-47.

[42] “SEGAN: SimVenture.” [Online]. Available: http://seriousgamesnet.eu/games/view/823. [Accessed: 22-Feb-2016].

[43] “PIXELearning.” [Online]. Available: /company/pixelearning. [Accessed: 28-May-2017]. 